\title{
Severe Dry Eye Disease - Facing the Treatment Challenges
}

\section{Proceedings of a Symposium Presented at the International Symposium on Ocular Pharmacology and Therapeutics} (ISOPT) 2014 in Reykjavik, Iceland, 20 June 2014

Expertly R eviewed by the Symposium Speakers:

Gysbert van Setten, ${ }^{1}$ Marc Labetoulle, ${ }^{2}$ Pasquale Aragona ${ }^{3}$ and Andrea Leonardi ${ }^{4}$

1. Associate Professor of Clinical Ophthalmology, St Eriks Eye Hospital, Karolinska Institutet, Stockholm, Sweden;

2. Professor of Ophthalmology, Ophthalmology Department, Bicêtre Hospital, Assistance Publique - Hôpitaux de Paris, South Paris University, France;

3. Professor of Ophthalmology, Head of the National Health System Referral Center for Ocular Surface Disease, University of Messina, Messina, Italy; 4. Assistant Professor of Clinical Ophthalmology, Department of Neuroscience, Ophthalmology Unit, University of Padua, Italy

\begin{abstract}
Dry eye disease (DED) is a multifactorial disease of the tears and ocular surface that results in symptoms of discomfort, visual disturbance and tear instability with potential damage to the ocular surface. It is often accompanied by hyperosmolarity of the tear film and inflammation of the ocular surface. The symposium 'Severe Dry Eye Disease - Facing the Treatment Challenges' held at the International Symposium on Ocular Pharmacology and Therapeutics (ISOPT) included four presentations from ophthalmological experts. The first presented a clinicalbased approach to facilitate the evaluation of DED in clinical practice and to provide ophthalmologists with straightforward tools to address objective signs of the disease and to alleviate subjective patients' symptoms. This was followed by a description of a stepwise approach to anti-inflammatory treatment of severe DED, highlighting the usefulness of topical ciclosporin for treating inflammatory conditions of the ocular surface such as severe DED. This was illustrated by the third speaker who presented the results of a clinical study (SICCANOVE) showing that, compared with vehicle, treatment with ciclosporin $0.1 \%$ yielded reduced corneal damage in patients with severe DED that appeared to be enhanced in patients with more severe keratitis. Last, a diagnostic algorithm of severe DED provided by a group of European experts (ODISSEY) was presented to facilitate important decision-making in advanced stages of the disease. The need for a consensus in diagnosis, terminology and therapy at a European and international level was emphasised. Aside from the intention to make diagnosis less complicated and technically advanced, the aspects of immunomodulatory treatment were addressed as an area for future research. Other future objectives in the treatment of DED include detection of possible precursor stages to offer the possibility of preventative care. Social economic effects are also important and increased international collaboration will be needed to address global inconsistencies in treatment.
\end{abstract}

\section{Keywords}

Dry eye disease (DED), ciclosporin, SICCANOVE study, corneal fluorescein staining (CFS), inflammation, ODISSEY

Disclosure: Gysbert van Setten, Marc Labetoulle, Pasquale Aragona and Andrea Leonardi have no conflicts of interest to declare.

Acknowledgements: Editorial assistance was provided by Catherine Amey at Touch Medical Media, London, UK.

Received: 17 July 2014 Accepted: 28 October 2014 Citation: European Ophthalmic Review, 2014;8(2):87-92 DOI: 10.17925/EOR.2014.08.02.87

Correspondence: Gysbert van Setten, St Eriks Eye Hospital, Karolinska Institutet, Polhemsgatan 50, 11282 Stockholm, Sweden. E: gysbert-botho.vansetten@sankterik.se

Support: The publication of this article was supported by Santen. The views and opinions expressed are those of the authors and not necessarily those of Santen.

\section{Pathogenetic Considerations and Implications on Simplified Decision-making in Dry Eye Disease}

\author{
Marc Labetoulle
}

Ophthalmology Department, Bicêtre Hospital, Assistance Publique - Hôpitaux de Paris, South Paris University, France

Dry eye disease (DED) is a multifactorial disease of the tears and ocular surface that results in symptoms of discomfort, visual disturbance and tear instability with potential damage to the ocular surface. It is accompanied by hyperosmolarity of the tear film and inflammation of the ocular surface. 1 The definition of DED, as outlined by the Dry Eye Workshop in 2007, combines objective findings with subjective symptoms and mechanistic considerations. Any abnormality of the ocular surface can trigger disequilibrium in all other components of tear dynamics resulting in a vicious circle with multiple entry points (see Figure 1). ${ }^{2}$ Eventually the patient develops a self-sustaining and, ultimately severe, DED. Thus, the objective in proposing a simplified 


\section{Figure 1: The Vicious Circle Theory of Dry Eye Disease $^{2}$}

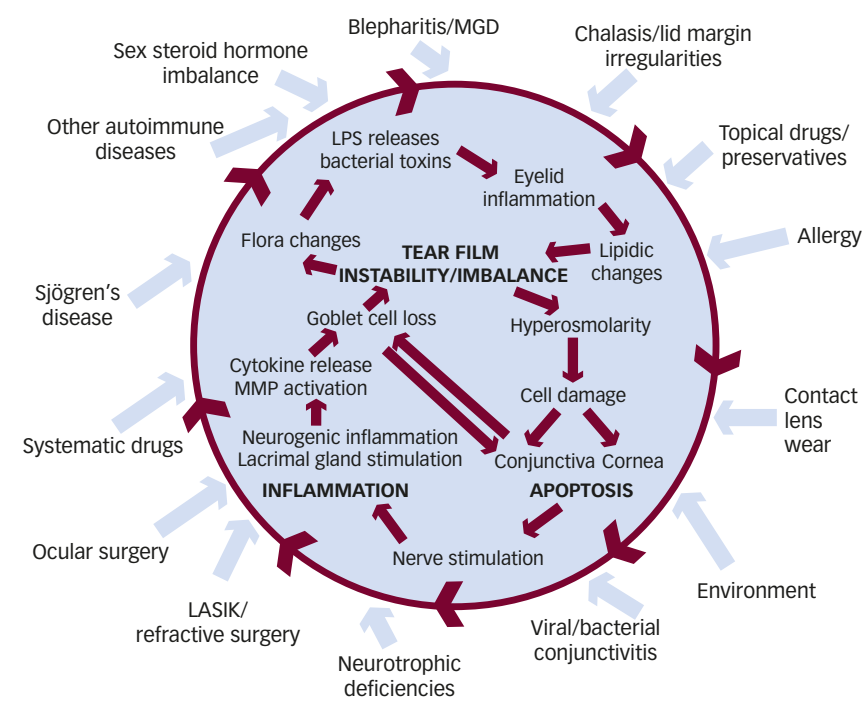

Modified from Baudouin 2007. ${ }^{2}$ LASIK = laser-assisted in-situ keratomileusis,

LPS = lipopolysaccharide; $M G D=$ meibomian gland disease;

$M M P=$ matrix metalloproteinase. epithelial damage, for which fluorescein staining can be informative. At this step, it is important to investigate any associated abnormalities such as conjunctivo-chalasis, follicles and papillae, meibomitis/blepharitis and reduction of corneal sensitivity. ${ }^{3}$

In the clinical-based approach proposed (see Figure 2), patients with DED fall into one of three conditions according to the type of correlation between subjective symptoms and objectives signs. In the first condition (where subjective symptoms appear more severe than objective signs), the main diagnosis to be considered is meibomian gland disease (MGD). ${ }^{4,5}$ Mood disorders may also account for the discrepancy between subjective symptoms and objective signs and there are also some DEDS that may involve sudden pain because of abnormalities in nerve endings into the superficial cornea leading to hyperesthesia. ${ }^{6}$

At the opposite end of the spectrum, there are patients with mild discomfort despite severe objective signs. The two main reasons for this are the presence of neurotrophic keratitis, of which post-viral is the most common type, and a long-stage DED, as we know that decreased sensitivity worsens with time in dry eyes.

The third and more often challenging type of DED patients are those with consistent symptoms and objectives signs.? The type of ocular

\section{Figure 2: The Simplified Decision-making Schema}

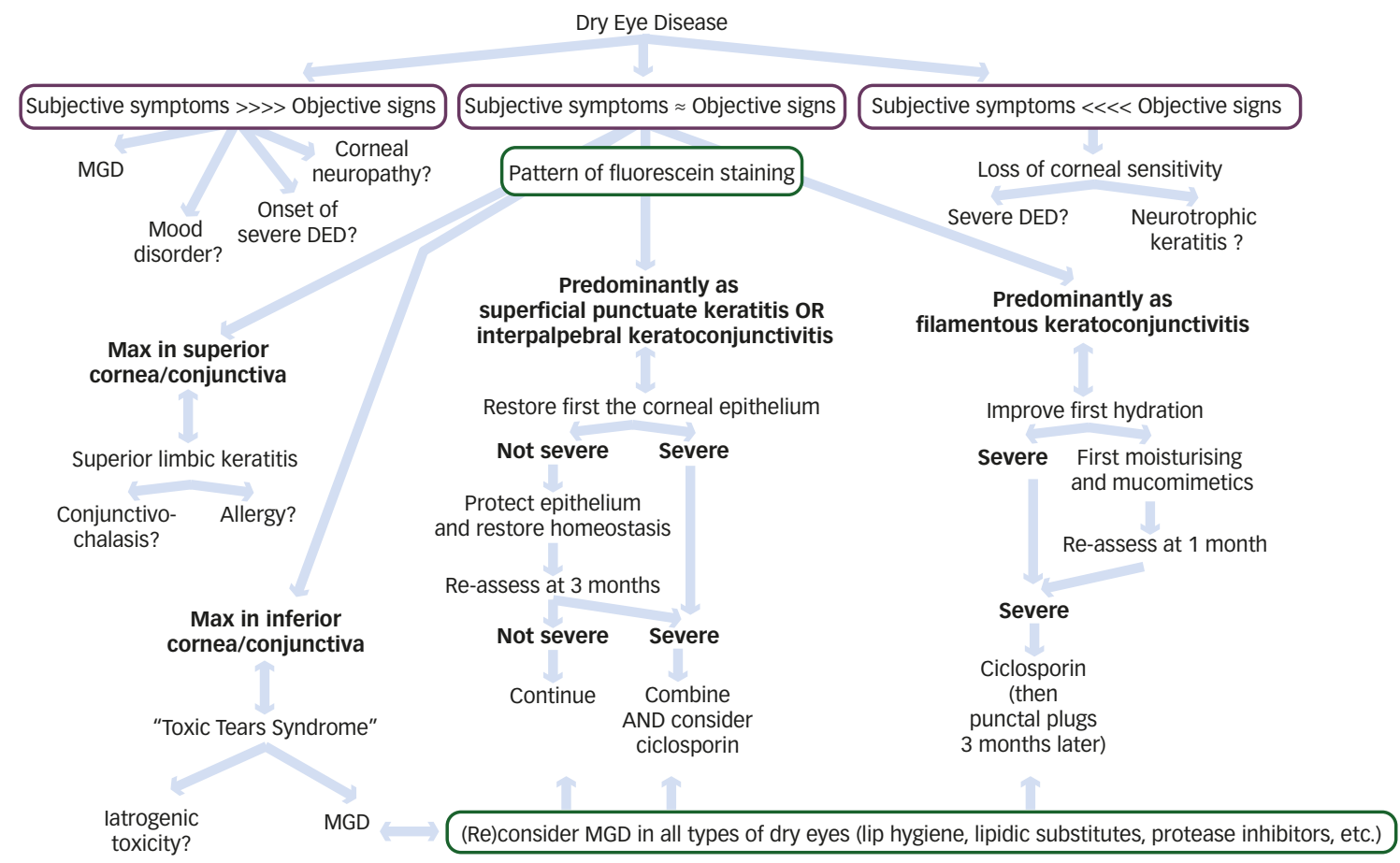

The physician may use different types of ophthalmic solutions according to the outcome of the previous step. Re-assessment and re-staging are vital to adapt the treatment according to the evolution of the dry eye disease (DED). Modified from Labetoulle and Baudouin. ${ }^{7}$ MGD $=$ meibomian gland disease.

decision-making schema is to assist ophthalmologists in daily practice in both choosing first-line treatment and adapting treatment for the long term.

First, it is important to assess spontaneous complaints and their frequency, duration and triggering factors. Spontaneous complaints can include: itching, burning, stinging, pain, soreness, sticky eyes, sensitivity to bright light/sunlight, sandy feeling or foreign body sensation, blurred vision, poor vision and intolerance to windy conditions and air conditioning and contact lenses. The second step is to examine corneal and conjunctival staining may aid the physician in setting up first-line treatment. When the maximum staining is located in the upper region of the cornea and superior conjunctiva, superior limbic keratitis is the most probable condition. It may be treated with lubricants and anti-inflammatory eye drops (short courses of steroids and/or ciclosporin). A maximum staining in the lower part of the eye is probably due to 'toxic tear syndrome' secondary either to MGD or toxic eye drops. Aetiological treatment will usually resolve this condition. If, however, the main staining is localised on the entire cornea, as a superficial punctuate keratitis, the initial treatment 
aim is to restore corneal epithelial quality. Maximum staining throughout the interpalpebral area, both on the cornea and on the conjunctiva, is suggestive of a chronic inflammation of the entire ocular surface, as observed in primary or secondary Sjögren disease. In these patients, it is important to provide osmoprotection as well as moisturising agents/ mucomimetics. If the first-line treatment does not improve the quality of the ocular surface and/or subjective symptoms after 3 months, longterm anti-inflammatory treatment, usually ciclosporin eye drops, are to be considered. The most aggressive condition is observed when filaments are present on the ocular surface. If after 1 month of lubricating treatment, there is no clear improvement, it is usually useful to set a chronic anti-inflammatory topical treatment (ciclosporin) with possible punctual plugging after re-assessment at 3 months.
By starting out from establishing the basic diagnosis of DED and then classifying it according to respective severity of signs and symptoms, this approach is complementing the work by the ODISSEY European Consensus Group, who have elaborated an algorithm that facilitates the diagnosis of severe DED even in the event of discordance between signs and symptoms.

Dry eye is a multifactorial disease of the tears and ocular surface that results in symptoms of discomfort, visual disturbance and tear instability, with potential damage to the ocular surface.

Dry eye is accompanied by increased osmolarity of the tear film and inflammation of the ocular surface.

\title{
Stepwise Approach to Anti-Inflammatory Treatment of Dry Eye Disease
}

\author{
Pasquale Aragona
}

Head of the National Health System Referral Center for Ocular Surface Disease, University of Messina, Messina, Italy

In the normal condition, the ocular surface system is able to modulate the immunological response to avoid negative consequences on its structures. If, however, pro-inflammatory stimuli are prolonged or strong enough, as occurs in severe DED, they can induce morphological and functional changes in the ocular surface, which can lead to the maintenance of chronic inflammation. ${ }^{9}$ Whatever the pathogenesis of the lacrimal dysfunction, the inflammatory process, once activated, is responsible for the maintaining of the ocular surface damage. Drugs blocking the inflammation cascade include corticosteroids, which have diffuse effects on both the adaptive and innate immune system and ciclosporin, which targets adaptive immunity. Ciclosporin treatment is associated with fewer unwanted side effects than those related to corticosteroid use and is considered an important tool for the treatment of ocular surface inflammation.

Ciclosporin can be useful in controlling chronic inflammation of the ocular surface since it has a mechanism of action that lowers T-cell activity as well as inhibiting apoptosis. Ciclosporin binds to cyclophillin to form a complex, which blocks the calcineurin from promoting the synthesis of inflammatory cytokines. Ciclosporin also blocks the transcription of the nuclear factor of activated T-cell, which would otherwise increase the activity of genes encoding for interleukin-2 and related cytokines. Its secondary activity prevents the mitochondrial permeability transition pore (MPTP) from opening thus inhibiting the release of cytochrome $\mathrm{C}$, a potent inducer of apoptosis.

In the therapeutic scheme for anti-inflammatory treatment of severe DED, proposed earlier this year (see Figure 3 ), ${ }^{10}$ ciclosporin treatment is initiated at diagnosis and it is advised to add corticosteroids starting at full dosage followed by a slow tapering, according to the clinical outcome, in a 2-month period. The rationale for this initial combined approach is based on existing data with ciclosporin $0.05 \%$ indicating that it achieves its best effect at 2 months of treatment. ${ }^{11}$ Corticosteroids are then tapered in accordance with clinical improvement and halted at 2 months.

Topical ciclosporin appears to be a very useful therapeutic tool for the treatment of inflammatory conditions of the ocular surface and is helpful in the treatment of dry eye.

Nevertheless, its use has been registered only in some countries outside Europe for treatment of dry eye disease (DED).

Further studies addressing these topics will shed light on the best way to use ciclosporin A and will render it, even more, an invaluable tool in the treatment of DED and other ocular surface inflammatory conditions.

\section{Figure 3: Proposed Therapeutic Schema for Anti-inflammatory Treatment of Severe Dry Eye}

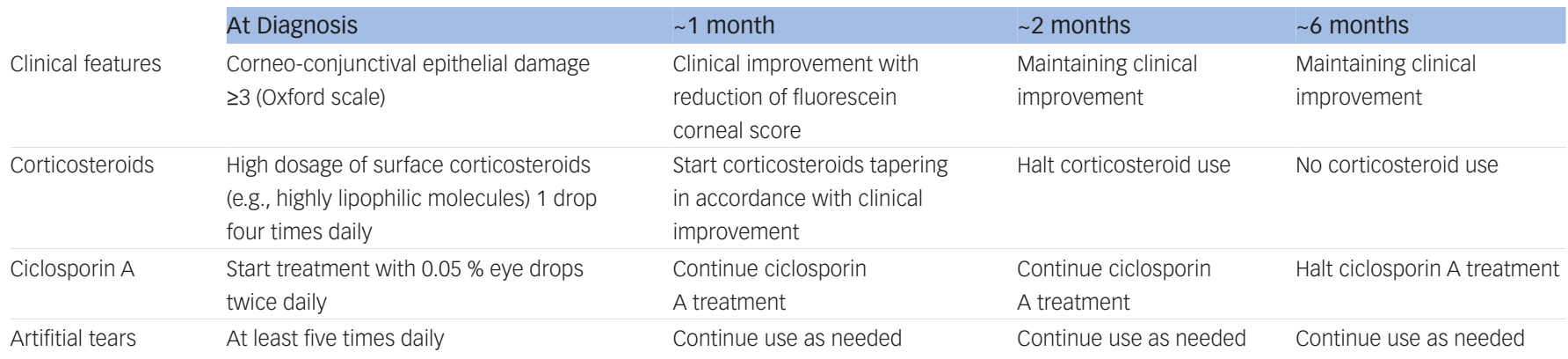

Possible recurrence of corneal damage will need further anti-inflammatory treatment with ciclosporin and possibly corticosteroids. 


\title{
Effects of Ciclosporin on Cornea Involvement in Patients with Severe Dry Eye
}

\author{
Andrea Leonardi
}

Department of Neuroscience, Ophthalmology Unit, University of Padua, Italy

\section{Figure 4: SICCANOVE Study Design in Patients with Severe Dry Eye Disease}

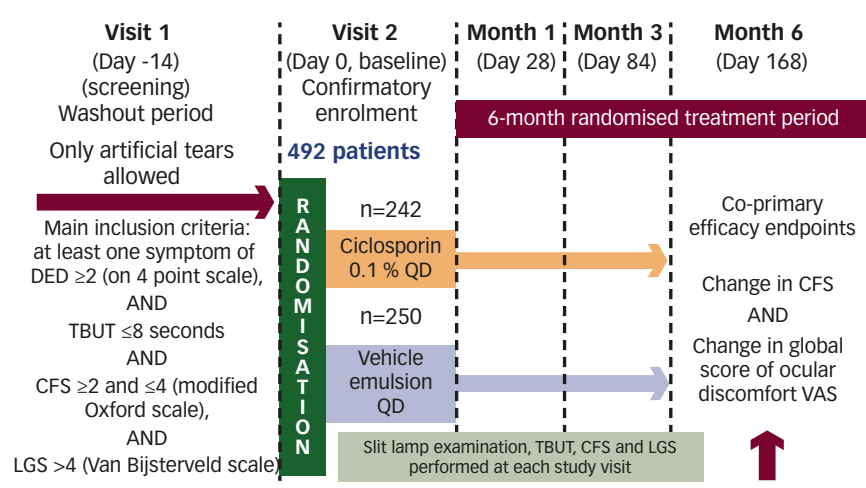

$C F S=$ corneal fluorescein staining; $D E D=$ dry eye disease; $L G S=$ Lissamine green staining; TBUT = tear break-up time; VAS = visual analogue scale.

Table 1: Ciclosporin $0.1 \%$ Shows the Greatest Treatment Benefit in Severe Dry Eye Disease Patients with the Highest Baseline Levels of Ocular Surface Inflammation in the SICCANOVE Study

\begin{tabular}{lll} 
CFS & CFS Responders at Month & CFS Responders at \\
Severity at & $6(\geq 2$ Grade Improvement) & Month $6(\geq 2$ Grade \\
Baseline & Ciclosporin 0.1\% & Improvement) Vehicle \\
Grade $2(n=178)$ & $21 \%$ & $12 \%$ \\
\hline Grade $3(n=226)$ & $37 \%$ & $31 \%$ \\
Grade $4(n=85)$ & $49 \% p=0.006^{*}$ & $20 \%$
\end{tabular}

${ }^{*} p$ value ciclosporin versus vehicle. A corneal fluorescein staining (CFS) responder is defined as patient with an improvement of 2 or more grades on the modified Oxford scale (CFS score).

Severe DED patients with severe keratitis have a raised risk of infection, vision loss and impaired quality of life. Still, there are no treatment guidelines for severe DED with severe keratitis. However, unapproved ciclosporin has been used in Europe for decades in such patients via hospital compounded formulations at various concentrations $(0.05 \%$, $0.5 \%, 1 \%$ or $2 \%$ ) and in various oils (e.g. olive or castor oil).

To address the unmet need for a topical ciclosporin therapy in the EU, Santen is developing a cationic emulsion of ciclosporin $0.1 \%$ for severe DED in adults with severe keratitis. This is an unpreserved oil-in-water cationic emulsion containing ciclosporin $1 \mathrm{mg} / \mathrm{ml}(0.1 \%)$. Its posology is 1 drop a day at bedtime. In animal models, the cationic emulsion of ciclosporin A $0.1 \%$ has been shown to lead to CSA corneal bioavailability that is four times higher than that of Restasis ${ }^{\circledR}$ (ciclosporin 0.05\%), ${ }^{12}$ giving way to the assumption that not only the concentration, but also the galenic formulation, contribute to effective delivery of ciclosporin to the epithelium.

The SICCANOVE study was a 6-month phase III, multicentre, randomised, controlled, double-masked study of ciclosporin $0.1 \%$ once daily versus vehicle enrolling 492 patients with dry eye signs (tear break-up time, $\leq 8$ second and corneal fluorescein staining [CFS], grade 2-4 [modified Oxford] and Schirmer test without anaesthesia $\geq 2$ and $<10 \mathrm{~mm} / 5$ minute and Lissamine green staining [LGS], [van Bijsterveld score $>4$ ] and $\geq 1$ dry eye symptom [ $\geq 2$ on a $0-4$ scale]). ${ }^{13}$ Corneal outcomes were evaluated at months 1, 3 and 6. The study design is outlined in Figure 4.

Ciclosporin $0.1 \%$ showed statistically significant improvement in CFS versus vehicle at month 1 (2.05 versus 2.25 on modified Oxford scale; $\mathrm{p}=0.002)$, month 3 ( 1.89 versus $2.06 ; \mathrm{p}=0.030$ ) and month 6 ( 1.65 versus $1.87 ; p=0.005)$.

Improvement was seen in DED symptoms, as measured by visual analogue scale (VAS), at months 1, 3 and 6 in both groups ( $\mathrm{p}=$ not significant [NS]). However, a poor correlation between DED signs and symptoms was shown in the SICCANOVE study ${ }^{14}$ with only $49 \%$ reporting improvement in both signs and symptoms. Improvement of symptoms despite no change (or worsening) of CFS was observed in $15 \%$, improvement of CFS but worsening of symptoms occurred in $19 \%$ and worsening of symptoms with no change (or worsening) of CFS was reported in $17 \%$.

A reduction in inflammation as shown in the expression of the inflammatory biomarker, HLA-DR, was seen with ciclosporin $0.1 \%$ in comparison with vehicle after 6 months $(p=0.022)$. Finally, post hoc analysis revealed greater improvements in ciclopsorin $0.1 \%$ treated patients over vehicle for corneal outcomes in groups with higher CFS scores at baseline (see Table 1)

The demonstrated correlation between HLA-DR expression and CFS in this study supports the important role of inflammation in the pathogenesis of DED and justifies the need for anti-inflammatory treatment in the management of DED.

Ciclosporin $0.1 \%$ is most effective in DED patients with greater ocular surface damage induced by chronic ocular surface inflammation.

Santen submitted a marketing authorisation application to the European Medicines Agency for the cationic emulsion of ciclosporin $0.1 \%$ in December 2013 via a centralised procedure. 


\title{
Present and Future Perspectives in the Treatment of Dry Eye Disease
}

\author{
Gysbert van Setten
}

St Eriks Eye Hospital, Karolinska Institutet, Stockholm, Sweden

Ocular surface disease with dry eye syndrome is an increasingly pressing concern in the everyday practice of ophthalmologists and the mismatch between symptoms and clinical findings often leaves the specialist with poor orientation to find the right therapy. The diagnosis is often demanding, difficult and rather time-consuming. In addition, the symptoms may be perceived as dramatic from the patient's perspective and the expectations for swift and accurate diagnosis as well as for successful treatment are high.

Today, the availability of the diagnostic ODISSEY algorithm facilitates important decision-making in advanced stages of DED. ${ }^{8}$ This algorithm is intended as a low-technology approach to identify the severest forms of DED combining one cardinal symptom and one decisive clinical sign (see Figure 5). However, among other factors, the classification of stages of dry eye or ocular surface diseases is still a problem. From various aspects of society and healthcare, the recognition as a disease and distinction to a mere complaint is an open issue.

Furthermore, simplified guidelines for follow-up need to be developed. such guidelines are needed for the monitoring of stages to attain and maintain success in treatment. In our understanding of DED, the precursor stages are a major focus for future investigation that may help specialists to progress from a treatment of a manifest disease to a preventative phase. However, the recognition of dry eye ocular surface disease as a disease more than a complaint does impact on the classification of stages and the access to treatment. Treatment, in turn, in its success and efficiency is dependent on accessibility and compliance. Accessibility and compensation for treatment are crucial topics since the group targeted by the disease is often the most vulnerable economically, and these factors impact on compliance. Global inconsistencies in accessibility to treatment need to be addressed. The real magnitude of socioeconomic effect of DED has become increasingly visible. However, prudence should be exerted when imposing the diagnosis DED. Exposed to sufficient environmental stress, each and every eye can show signs of surface dryness and DED - without reflecting a disease as such. Provoked DED does outline the threshold of maximal oculo-dacryological compensation capacity - it does not necessarily reflect natural conditions of environment and its effect in the pathophysiology of dry eye genesis. The increased number of observations and diagnoses of DED in context with computer work or other environmental factors must be evaluated carefully.

Other future objectives in the treatment of DED include an optimised, adaptive and flexible treatment with different agents and components. There are the aims of meeting the demand of balancing of viscosity (see Figure 6) as well as optimised timing and dosage of immune-modulatory therapies; aside this, the creation of improved microenvironments, definition and improved management of risk environments.

Development of patient education as well as coaching is a major focus for the future. Parallel to this, enhanced efforts

\section{Figure 5: Consensus Derived Scoring Algorithm for Severe DED Diagnosis}

Preliminary step: Establish DED diagnosis with tear test (i.e. TBUT)

Step 1: Primary criteria for severe DED diagnosis Symptoms and signs: OSDI $\geq 33$ and CFS Oxford Scale $\geq 3$ ?

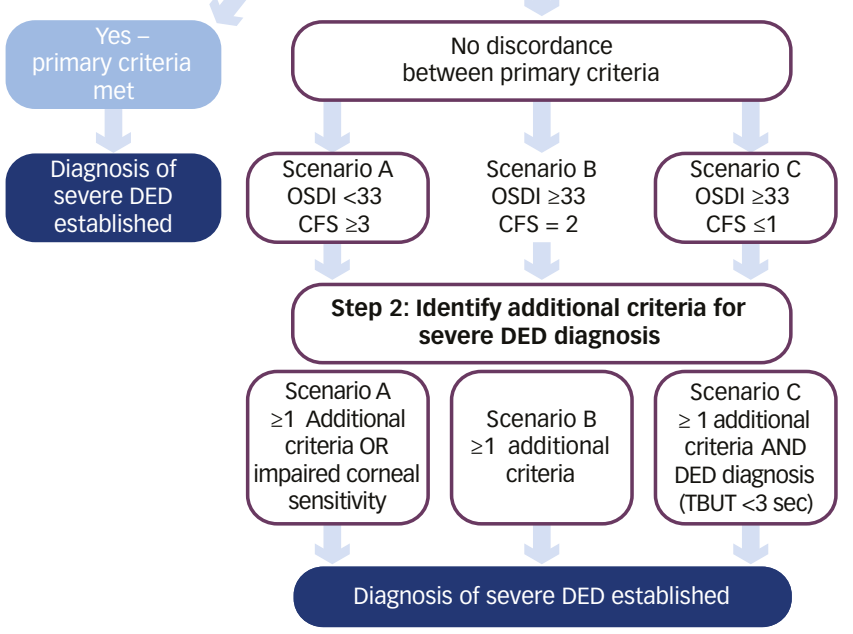

$C F S=$ corneal fluorescein staining; $D E D=$ dry eye disease $; O S D I=$ Ocular surface Disease Index; TBUT = tear break-up time. Modified from Baudouin et al. ${ }^{9}$

\section{Figure 6: Balancing Viscosity-Drops/Gels/ Ointments}

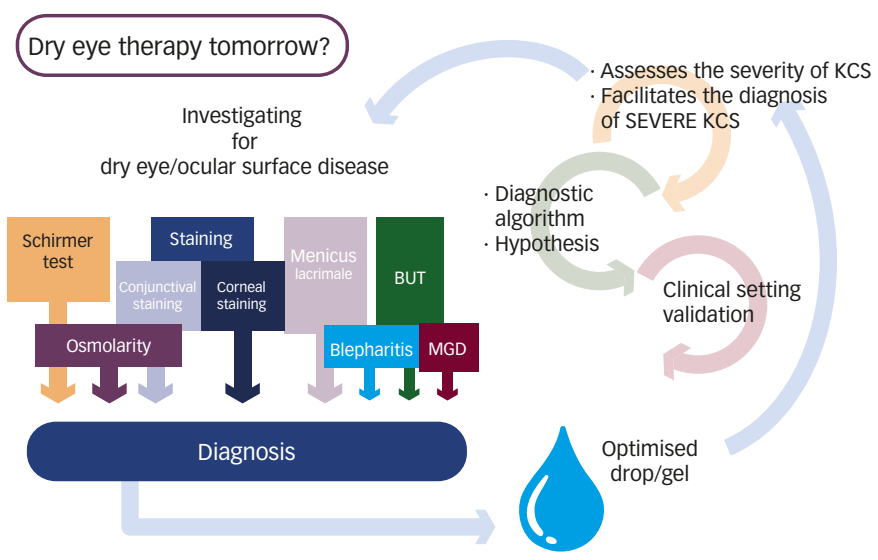

$B U T=$ break-up time; $K C S=$ keratoconjunctivitis sicca; $M G D=$ meibomian gland disease.

for healthcare professional educational programmes will be increasingly important.

Finally, efforts can never be enough to reach consensus on treatment and diagnosis. These efforts need to be extended to a global scale, which will require increased international collaboration. 
1. International Dry Eye WorkShop, The definition and classification of dry eye disease: report of the Definition and Classification Subcommittee of the International Dry Eye WorkShop (2007), Ocul Surf, 2007;5:75-92.

2. Baudouin C, [A new approach for better comprehension of diseases of the ocular surface]. [Article in French], $\mathrm{fFr}$ ophtalmol, 2007;30:239-46.

3. International Dry Eye WorkShop. Methodologies to diagnose and monitor dry eye disease: report of the Diagnostic Methodology Subcommittee of the International Dry Eye Methodology Subcommittee of the Internation
WorkShop (2007), Ocul Surf, 2007; 5:108-52.

WorkShop (2007), Ocul Surf, 2007;5:108-52.
4. Lemp MA, Crews LA, Bron AJ, et al., Distribution of aqueousdeficient and evaporative dry eye in a clinic-based patient cohort: a retrospective study Cornea, 2012;31:472-8.

5. Nichols KK, Nichols JJ, Mitchell GL, The lack of association between signs and symptoms in patients with dry eye disease, Cornea, 2004;23:762-70

6. Rosenthal P, Borsook D, The corneal pain system. Part I: the missing piece of the dry eye puzzle, Ocul Surf, 2012;10:2-14.

7. Labetoulle M, Baudouin C, From pathogenic considerations to a simplified decision-making schema in dry eye disease, $J$ Fr Ophtalmol, 2013:36:543-7.

8. Baudouin C, Aragona P, Van Setten G, et al., Diagnosing the severity of dry eye: a clear and practical algorithm, $\mathrm{Br}$ J Ophthalmol, 2014;98:1168-76.

9. Aragona P, Rania L, Ruzzolo D, In: Ocular Surface Disease, Benitez-del-Castillo JM, Michael A Lemp (eds), London: JP Medical Ltd, 2013

10. Aragona P, Topical cyclosporine: are all indications justified? Br J Ophthalmol, 2014;98:1001-2.

11. Chung YW, Oh TH, Chung SK, The effect of topical cyclosporine $0.05 \%$ on dry eye after cataract surgery, Korean
J Ophthalmol, 2013;27:167-71.

12. Daull P, Lallemand F, Philips B, et al., Distribution of cyclosporine A in ocular tissues after topical administration of cyclosporine A cationic emulsionsto pigmented rabbits, Cornea, 2013;32:345-54.

13. Buggage,RR Amrane M, Ismail, D, et al., The effect of Cyclokat ${ }^{\circledR}$ (unpreserved $0.1 \%$ cyclosporine cationic emulsion) on corneal involvement in patients with moderate to severe dry eye disease participating in a phase III, multicenter, randomized, controlled, double-masked, clinical trial, Eur 」 randomized, controlled, double-masked, clinical trial, eur

14. Lemp MA, Baudouin C, Amrane M, et al., Poor correlation between dry eye disease (DED) signs and symptoms in a phase
betring iii randomized clinical trial. Poster presented at the Association for Research in Vision and Ophthalmology (ARVO) 2011, (Fort Lauderdale, Florida, USA, 1-5 May 2011) on 3 May 2011 